\title{
Phenylvinylidene Clusters Containing \\ ReFePt Metal Cores \\ and Chelate Diphosphine Ligands \\ at the Platinum Atom
}

\author{
Victor V. Verpekin*a, Alexander A. Kondrasenko ${ }^{a}$, \\ Ruslan O. Ergaev ${ }^{b}$, Oleg S. Chudin \\ Nina I. Pavlenko and Anatoly I. Rubaylo ${ }^{\mathrm{a}, \mathrm{b}}$ \\ anstitute of Chemistry and Chemical Technology SB RAS \\ FRC "Krasnoyarsk Science Center SB RAS" \\ 50/24 Akademgorodok, Krasnoyarsk, 660036, Russia \\ ${ }^{b}$ Siberian Federal University \\ 79 Svobodny, Krasnoyarsk, 660041, Russia
}

Received 21.02.2017, received in revised form 08.04.2017, accepted 31.05.2017

\begin{abstract}
Two different synthetic methods were applied to obtain heteronuclear phenylvinylidene clusters $\mathrm{CpReFePt}\left(\mu_{3}-\mathrm{C}=\mathrm{CHPh}\right)(\mathrm{CO})_{5}(\mathrm{P}-\mathrm{P})(\mathrm{P}-\mathrm{P}-$ bis(diphenylphosphino)ethane (1), bis(diphenylphosphino) propane (2)). The compounds were studied by IR and NMR spectroscopy. Their molecular structures were proposed based on IR, NMR spectroscopic data.
\end{abstract}

Keywords: rhenium, platinum, iron, cluster, vinylidene, heterometallic complexes, vinylidene complexes.

DOI: $10.17516 / 1998-2836-0021$.

(C) Siberian Federal University. All rights reserved

* Corresponding author E-mail address:vvv@sany-ok.ru 


\title{
Фенилвинилиденовые кластеры \\ с остовом ReFePt, \\ содержащие хелатные дифосфиновые лиганды \\ при атоме платины
}

\author{
В.В. Верпекин ${ }^{\mathrm{a}}$, А.А. Кондрасенко ${ }^{\mathrm{a}}$, Р. О. Ергаев \\ О.С. Чудин ${ }^{\text {, Н.И. Павленко }}{ }^{\text {, А.И. Рубайло }}{ }^{\text {, }, ~}$ \\ ${ }^{a}$ Институт химии и химической технологии СО РАН \\ ФИЦ «Красноярский научный центр СО РАН» \\ Россия, 660036, Красноярск, Академгородок, 50/24 \\ ${ }^{6}$ Сибирский федеральный университет \\ Россия, 660041, Красноярск, пр. Свободнылй, 79
}

Двумя различными способами синтезированы гетеротрехъядерные фенилвинилиденовые кластеры типа CpReFePt $\left(\mu_{3}-C=C H P h\right)(C O)_{5}(P-P)$ (где P-P - бис(дифенилфосфино)этан (1) или бис(дифенилфосфино)пропан (2)). Полученные соединения изучены методами ИКи ЯМР-спектроскопии. На основе спектроскопических данных предложено строение кластеров.

Ключевые слова: рений, платина, железо, кластеры, винилиден, гетерометаллические комплексы, винилиденовые комплексы.

\section{Введение}

Исследования структуры, физико-химических свойств и закономерностей образования полиметаллических соединений с различными органическими лигандами вносят вклад в фундаментальные разделы современной химии, такие как теория строения и реакционной способности, теория процессов каталитического синтеза [1-6]. Наибольший интерес вызывают исследования гетерометаллических кластеров и комплексов, в которых непредельные карбеновые лиганды координированы с несколькими атомами металлов [7-9].

Для получения кластерных гетерометаллических соединений используют в основном превращения органических молекул и лигандов на заранее сформированном металлическом центре в жестких условиях [10]. Однако такие методы индивидуальны для каждого случая, что не позволяет прогнозировать состав и строение конечного продукта.

Наиболее эффективный подход к получению гетерометаллических кластеров основан на способности моно- и биядерных гетерометаллических комплексов, содержащих непредельные углеводородные лиганды, селективно присоединять координационно- и электронноненасыщенные металлосодержащие фрагменты [11-16]. Данный подход успешно реализован нами для синтеза би- и трехъядерных гетерометаллических винилиденовых комплексов и кластеров [14, 17-19]. 
Несмотря на многообразие гетерометаллических $\mu_{3}$-винилиденовых кластеров в настоящее время известно лишь небольшое число таких соединений, содержащих атом рения. Первый $\mu_{3}$-винилиденовый кластер, содержащий атом рения $\mathrm{CpReFePt}\left(\mu_{3}-\mathrm{C}=\mathrm{CHPh}\right)(\mathrm{CO})_{6}\left(\mathrm{PPh}_{3}\right)(3)$, был получен относительно недавно, в 2009 г. [20], позже синтезированы и охарактеризованы кластер $\mathrm{CpReFePt}\left(\mu_{3}-\mathrm{C}=\mathrm{CHPh}\right)(\mathrm{CO})_{5}\left[\mathrm{P}\left(\mathrm{OPr}^{\mathrm{i}}\right)_{3}\right]_{2}$ (4) и кластеры, содержащие металлический остов $\mathrm{ReFePd}$ и хелатные дифосфиновые лиганды при атоме палладия, $\mathrm{CpReFePd}\left(\mu_{3}-\mathrm{C}=\mathrm{CHPh}\right)(\mathrm{CO})_{5}(\mathrm{P}-\mathrm{P})$ (P-P = dppe (5), dppp (6)) [21, 22]. Поэтому поиск новых путей синтеза, изучение закономерностей образования и свойств гетерометаллических винилиденовых комплексов рения является актуальным. В этой связи нами изучена возможность синтеза и свойства $\mu_{3}$-винилиденовых кластеров с остовом ReFePt и хелатными дифосфиновыми лигандами у атома платины.

\section{Экспериментальная часть}

Все операции по синтезу и выделению комплексов проводили в атмосфере аргона. Растворители (бензол, диэтиловый эфир, петролейный эфир, тетрагидрофуран, хлористый метилен) предварительно очищали от примесей, следов воды и кислорода путем перегонки над соответствующими осушителями. Комплексы $\mathrm{Cp}(\mathrm{CO})_{2} \operatorname{Re}(\mu-\mathrm{C}=\mathrm{CHPh}) \mathrm{Pt}(\mathrm{P}-\mathrm{P})(\mathrm{P}-\mathrm{P}=\mathrm{dppe}(7)$; dppp (8)) и $\mathrm{CpReFePt}\left(\mu_{3}-\mathrm{C}=\mathrm{CHPh}\right)(\mathrm{CO})_{6}\left(\mathrm{PPh}_{3}\right)$ (3) синтезированы по методикам [20, 23]. В экспериментах использованы 1,2-бис(дифенилфосфино)этан (Merck), 1,3-бис(дифенилфосфино)пропан (Acros). Нонакарбонил дижелеза получали по методике [24].

Спектры ИК измеряли на ИК-Фурье-спектрометре Tensor 27 (Bruker, Германия). Спектры ЯMP ${ }^{1} \mathrm{H},{ }^{13} \mathrm{C}$ и ${ }^{31}$ Р регистрировали на Фурье-спектрометре ЯMP AVANCE III 600 (Bruker, Германия). Химические сдвиги в спектрах были определены относительно остаточных протонов дейтерированных растворителей и приведены в миллионных долях (м.д.). Константы спинспиновых взаимодействий указаны в герцах (Гц).

Кластеры $\mathrm{CpReFePt}\left(\mu_{3}-\mathrm{C}=\mathrm{CHPh}\right)(\mathrm{CO})_{5}(\mathrm{dppe})(\mathbf{1})$ и $\mathrm{CpReFePt}\left(\mu_{3}-\mathrm{C}=\mathrm{CHPh}\right)(\mathrm{CO})_{5}(\mathrm{dppp})(\mathbf{2})$ синтезированы по следующим методикам:

Синтез $\mathrm{CpReFePt}\left(\mu_{3}-\mathrm{C}=\mathrm{CHPh}\right)(\mathrm{CO})_{5}(\mathrm{dppe})(\mathbf{1})$

Метод 1. К раствору 0,079 г (0,079 ммоль) $\mathrm{Cp}(\mathrm{CO})_{2} \operatorname{Re}(\mu-\mathrm{C}=\mathrm{CHPh}) \operatorname{Pt}(\mathrm{dppe})$ (7) в 10 мл бензола при интенсивном перемешивании в атмосфере аргона добавляли 0,121 г (0,33 ммоль) $\mathrm{Fe}_{2}(\mathrm{CO})_{9}$. Реакционную смесь перемешивали в течение 1 ч, фильтровали через канюль, затем упаривали в вакууме. Остаток растворяли в бензоле и наносили на колонку $\mathrm{Al}_{2} \mathrm{O}_{3}(5 \times 2$ см). Колонку элюировали смесью гексан-бензол (7:3) и бензолом. В первой темно-красной зоне после удаления растворителей, содержалось 0,048 г (53 \%) $\mathrm{CpReFePt}\left(\mu_{3}-\mathrm{C}=\mathrm{CHPh}\right)(\mathrm{CO})_{5}(\mathrm{dppe})(\mathbf{1})$. Из второй желтой зоны выделили 0,032 г (40 \%) исходного комплекса $\mathrm{Cp}(\mathrm{CO})_{2} \operatorname{Re}(\mu-\mathrm{C}=\mathrm{CHPh})$ Pt(dppe) (7).

Метод 2. К раствору 0,061 г (0,059 ммоль) $\mathrm{CpReFePt}\left(\mu_{3}-\mathrm{C}=\mathrm{CHPh}\right)(\mathrm{CO})_{6}\left(\mathrm{PPh}_{3}\right)(3)$ в 10 мл бензола в инертной атмосфере добавляли 0,028 г (0,070 ммоль) dpре. Реакционную смесь перемешивали в течение 2 ч, затем концентрировали при пониженном давлении до 1 мл и разбавили 10 мл гексана, образовался ярко-красный осадок. С выпавшего осадка декантировали маточный раствор, осадок промывали гексаном $3 \times 2$ мл и высушивали в вакууме. Получили 0,059 г (87 \%) комплекса $\mathrm{CpReFePt}\left(\mu_{3}-\mathrm{C}=\mathrm{CHPh}\right)(\mathrm{CO})_{5}(\mathrm{dppe})(\mathbf{1})$, идентифицированного по ИК-спектрам. 
ИК-спектр $\left(\mathrm{CH}_{2} \mathrm{Cl}_{2}, \mathrm{~cm}^{-1}\right): 1996,1928,1906,1842$ (v(CO)); $\left(\mathrm{KBr}, \mathrm{cm}^{-1}\right): 1994,1926,1902,1843$ $(v(\mathrm{CO}))$.

В спектрах ЯМР ${ }^{1} \mathrm{H}$ соотношение сигналов циклопентадиенильного лиганда двух изомеров составляет $3: 1$.

1 изомер:

${ }^{13} \mathrm{C}\left\{{ }^{1} \mathrm{H}\right\}$ ЯМР $\left(\mathrm{CD}_{2} \mathrm{Cl}_{2}\right) \delta: 31,9$ (дд, $\left.-\mathrm{CH}_{2^{-}}, J_{\mathrm{CP}}=36, J_{\mathrm{CP}}=12\right) ; 33,2$ (дд, $-\mathrm{CH}_{2^{-}}, J_{\mathrm{CP}}=20, J_{\mathrm{CP}}=19$ ); 90,1 (c, $\left.\mathrm{C}_{5} \mathrm{H}_{5}\right) ; 96,6$ (д, $\left.{ }^{3} J_{\mathrm{CP}}=29, \mathrm{C}^{1}=\underline{\mathrm{C}}^{2} \mathrm{HPh}\right) ; 125-137(-\mathrm{Ph}) ; 144,4\left(\mathrm{C}_{\text {кл }}=\mathrm{C}^{2} \mathrm{H}_{\mathrm{C}_{6}} \mathrm{H}_{5}\right) ; 209,3$ (c, Re- $\left.\underline{\mathrm{CO}}\right)$; 211,6 (c, Re- $\underline{\mathrm{CO}}) ; 263,9\left(\mu-\mathrm{C}^{1}, J_{\mathrm{CP}}=4\right)$.

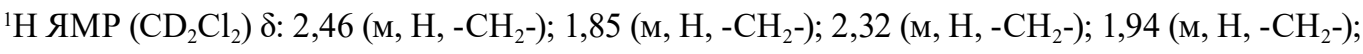
$5,13\left(\mathrm{c}, 5 \mathrm{H}, \mathrm{C}_{5} \mathrm{H}_{5}\right) ; 7,01$ (д, H, $\left.J_{\mathrm{PtP}}=29 ;{ }^{4} J_{\mathrm{HP} 1}=7,7,{ }^{4} J_{\mathrm{HP} 2}=11,67,=\mathrm{C}^{2} \underline{\mathrm{HPh}}\right) ; 6,2-7,8(25 \mathrm{H},-\mathrm{Ph})$.

${ }^{31} \mathrm{P}\left\{{ }^{1} \mathrm{H}\right\}$ ЯMP $\left(\mathrm{CD}_{2} \mathrm{Cl}_{2}\right) \delta: 52,56\left(\mathrm{P}_{1}, J_{\mathrm{PP}}=7,08, J_{\mathrm{PtP}}=3300\right) ; 52,00\left(\mathrm{P}_{2}, J_{\mathrm{PP}}=7,08, J_{\mathrm{PtP}}=3600\right)$.

2 изомер:

${ }^{13} \mathrm{C}\left\{{ }^{1} \mathrm{H}\right\}$ ЯМР $\left(\mathrm{CD}_{2} \mathrm{Cl}_{2}\right) \delta: 32,5\left(\mathrm{м},-\mathrm{CH}_{2}\right) ; 29,8\left(\mathrm{м},-\mathrm{CH}_{2}-\right) ; 89,4\left(\mathrm{c}, \mathrm{C}_{5} \mathrm{H}_{5}\right) ; 96,3$ (д, ${ }^{3} \mathrm{JP}_{\mathrm{CP}}=28$, $\left.\mathrm{C}^{\mathrm{l}}=\underline{\mathrm{C}}^{2} \mathrm{HPh}\right) ; 125-137(-\mathrm{Ph}) ; 142,2\left(\mathrm{C}_{\text {кл }}=\mathrm{C}^{2} \mathrm{HC}_{6} \mathrm{H}_{5}\right) ; 207,4$ (c, Re- $\left.\underline{\mathrm{CO}}\right) ; 209,1$ (c, Re- $\left.\underline{\mathrm{CO}}\right) ; 255,1\left(\mu-\mathrm{C}^{1}\right)$.

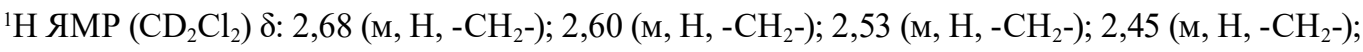
5,37 (c, 5H, $\left.\mathrm{C}_{5} \mathrm{H}_{5}\right) ; 6,2-7,8$ (25H, -Ph); 7,11 (д, $\mathrm{H},{ }^{4} J_{\mathrm{HP1}}=7,7,=\mathrm{C}^{2} \underline{\mathrm{HPh}}$ );

${ }^{31} \mathrm{P}\{1 \mathrm{H}\}$ ЯMP $\left(\mathrm{CD}_{2} \mathrm{Cl}_{2}\right) \delta: 48,76\left(\mathrm{P}_{1}, J_{\mathrm{PP}}=11,6, J_{\mathrm{PtP}}=3300\right) ; 49,9\left(\mathrm{P}_{2}, J_{\mathrm{PP}}=11,6, J_{\mathrm{PtP}}=3500\right)$.

Синтез $\mathrm{CpReFePt}\left(\mu_{3}-\mathrm{C}=\mathrm{CHPh}\right)(\mathrm{CO})_{5}(\mathrm{dppp})(2)$

Метод 1. К раствору 0,162 г (0,159 ммоль) $\mathrm{Cp}(\mathrm{CO})_{2} \operatorname{Re}(\mu-\mathrm{C}=\mathrm{CHPh}) \operatorname{Pt}(\mathrm{dppp})(\mathbf{8})$ в 10 мл бензола в инертной атмосфере добавляли 0,236 г (0,648 ммоль) $\mathrm{Fe}_{2}(\mathrm{CO})$. Реакционную смесь перемешивали в течение 1 ч, фильтровали через канюль, а затем упаривали на водоструйном насосе. Остаток растворяли в бензоле и наносили на колонку $\mathrm{Al}_{2} \mathrm{O}_{3}(5 \times 2 \mathrm{~cm})$. Колонку элюировали смесью гексан-бензол (6:4) и бензолом. В первой темно-красной зоне, после удаления растворителей, содержалось 0,057 г (31 \%) $\mathrm{CpReFePt}\left(\mu_{3}-\mathrm{C}=\mathrm{CHPh}\right)(\mathrm{CO})_{5}(\mathrm{dppp})(2)$. Из второй желтой зоны выделили 0,075 г (46 \%) исходного комплекса $\mathrm{Cp}(\mathrm{CO})_{2} \operatorname{Re}(\mu-\mathrm{C}=\mathrm{CHPh}) \mathrm{Pt}(\mathrm{dppp})(\mathbf{8})$.

Метод 2. К раствору 0,053 г (0,051 ммоль) $\mathrm{CpReFePt}\left(\mu_{3}-\mathrm{C}=\mathrm{CHPh}\right)(\mathrm{CO})_{6}\left(\mathrm{PPh}_{3}\right)(3)$ в 10 мл бензола в инертной атмосфере добавляли 0,025 г (0,06 ммоль) dppp. Реакционную смесь перемешивали в течение 2 ч, затем концентрировали при пониженном давлении до 1 мл и разбавляли 10 мл гексана, образовался ярко-красный осадок. С выпавшего осадка декантировали маточный раствор, осадок промывали гексаном $3 \times 2$ мл и высушивали в вакууме. Получили 0,049 г (83\%) комплекса $\mathrm{CpReFePt}\left(\mu_{3}-\mathrm{C}=\mathrm{CHPh}\right)(\mathrm{CO})_{5}(\mathrm{dppp})(2)$, идентифицированного по ИК-спектрам.

ИК-спектр (КВr, см-1): 1996, 1928, 1905, 1845 (v(СО)).

В спектрах ЯМР ${ }^{1} \mathrm{H}$ соотношение сигналов циклопентадиенильного лиганда двух изомеров составляет 1:1.

1 изомер:

${ }^{1} \mathrm{H}$ ЯMP $\left(\mathrm{CD}_{2} \mathrm{Cl}_{2}\right) \delta: 1,55-1,85\left(\mathrm{м}, 6 \mathrm{H},-\left(\mathrm{CH}_{2}\right)_{3}\right)$ ); 4,95 (c, 5H, $\left.\mathrm{C}_{5} \mathrm{H}_{5}\right) ; 7,00$ (дд, $\mathrm{H}, J_{\mathrm{CP}}=10,6$, $\left.J_{\mathrm{CP}}=8,6, J_{\mathrm{PtH}}=51,=\mathrm{C}^{2} \underline{\mathrm{HPh}}\right) ; 6,7-7,69(25 \mathrm{H},-\mathrm{Ph})$.

${ }^{31} \mathrm{P}\left\{{ }^{1} \mathrm{H}\right\}$ ЯMP $\left(\mathrm{CD}_{2} \mathrm{Cl}_{2}\right) \delta: 1,52\left(\mathrm{P}_{1}, J_{\mathrm{PP}}=34,7, J_{\mathrm{PtP}}=3231\right) ;-0,77\left(\mathrm{P}_{2}, J_{\mathrm{PP}}=34,7, J_{\mathrm{PtP}}=3488\right)$.

2 изомер:

${ }^{1} \mathrm{H}$ ЯMP $\left(\mathrm{CD}_{2} \mathrm{Cl}_{2}\right) \delta: 5,35$ (c, 5H, $\left.\mathrm{C}_{5} \mathrm{H}_{5}\right) ; 7,11$ (д, $\mathrm{H}, J_{\mathrm{CP}}=4$, = $\left.\mathrm{C}^{2} \underline{\mathrm{HPh}}\right) ; 6,7-7,69$ (25H, - $\left.\mathrm{Ph}\right)$. ${ }^{31} \mathrm{P}\left\{{ }^{1} \mathrm{H}\right\}$ ЯMP $\left(\mathrm{CD}_{2} \mathrm{Cl}_{2}\right) \delta:-0,43\left(\mathrm{P}_{1}, J_{\mathrm{PP}}=35,6, J_{\mathrm{PtP}}=3449\right) ;-1,54\left(\mathrm{P}_{2}, J_{\mathrm{PP}}=35,6, J_{\mathrm{PtP}}=3125\right)$. 


\section{Результаты и обсуждение}

Ранее на основе реакций биядерных комплексов $\mathrm{Cp}(\mathrm{CO})_{2} \mathrm{Mn}(\mu-\mathrm{C}=\mathrm{CHPh}) \mathrm{Pt}(\mathrm{L})\left(\mathrm{L}^{\prime}\right)$ $\left(\mathrm{L}=\mathrm{L}^{\prime}=\mathrm{PPh}_{3}, \mathrm{P}\left(\mathrm{OPr}^{\mathrm{i}}\right)_{3}, \mathrm{P}(\mathrm{OEt})_{3} ; \mathrm{L}=\mathrm{PPh}_{3}, \mathrm{~L}^{\prime}=\mathrm{CO}\right)$ с $\mathrm{Fe}_{2}(\mathrm{CO})_{9}$ в нашей группе была синтезирована серия трехъядерных $\mu_{3}$-винилиденовых кластеров $\mathrm{CpMnFePt}\left(\mu_{3}-\mathrm{C}=\mathrm{CHPh}\right)(\mathrm{CO})_{6}(\mathrm{~L})$, содержащих только терминальные фосфорсодержащие и карбонильные лиганды при атоме платины $[25,26]$. Однако реакции биядерных винилиденовых MnPt комплексов, содержащих хелатные дифосфиновые лиганды с нонакарбонилом дижелеза приводили к отщеплению циклопентадиенилатрикарбонил марганца и образованию продуктов переметаллирования исходных соединений (рис. 1) [27].

Только при реакции комплекса $\mathrm{Cp}(\mathrm{CO})_{2} \mathrm{Mn}(\mu-\mathrm{C}=\mathrm{CHPh}) \mathrm{Pt}(\mathrm{dppm})$ с $\mathrm{Fe}_{2}(\mathrm{CO})_{9}$ среди продуктов реакции был получен $\mu_{3}$-винилиденовый кластер $\mathrm{CpMnFePt}\left(\mu_{3}-\mathrm{C}=\mathrm{CHPh}\right)(\mathrm{CO})_{5}(\mu-\mathrm{dppm})(\mathbf{9})$ с выходом 46 \%, в котором dppm лиганд образовывал мостик между железо- и платинасодержащими фрагментами (рис. 1) [28]. Для ренийсодержащих комплексов $\mathrm{Cp}(\mathrm{CO})_{2} \operatorname{Re}(\mu-\mathrm{C}=\mathrm{CHPh})$ $\mathrm{Pd}(\mathrm{dppe})$ и $\mathrm{Cp}(\mathrm{CO})_{2} \operatorname{Re}(\mu-\mathrm{C}=\mathrm{CHPh}) \operatorname{Pd}(\mathrm{dppp})$ было показано, что их взаимодействие с $\mathrm{Fe}_{2}(\mathrm{CO})_{9}$ приводит к образованию трехъядерных $\mu_{3}$-винилиденовых кластеров $\mathrm{CpReFePd}\left(\mu_{3}-\mathrm{C}=\mathrm{CHPh}\right)$ (dppe) (5) и $\mathrm{CpReFePd}\left(\mu_{3}-\mathrm{C}=\mathrm{CHPh}\right)(\mathrm{dppp})(\mathbf{6})$, однако в реакционной смеси образуются и другие продукты за счет взаимодействия образовавшихся кластеров 5 и 6 с Fе $2(\mathrm{CO})_{9}$ [21].

Для установления возможности получения гетерометаллических трехъядерных $\mu_{3}$-винилиденовых кластеров, содержащих при атоме платины хелатные дифосфиновые

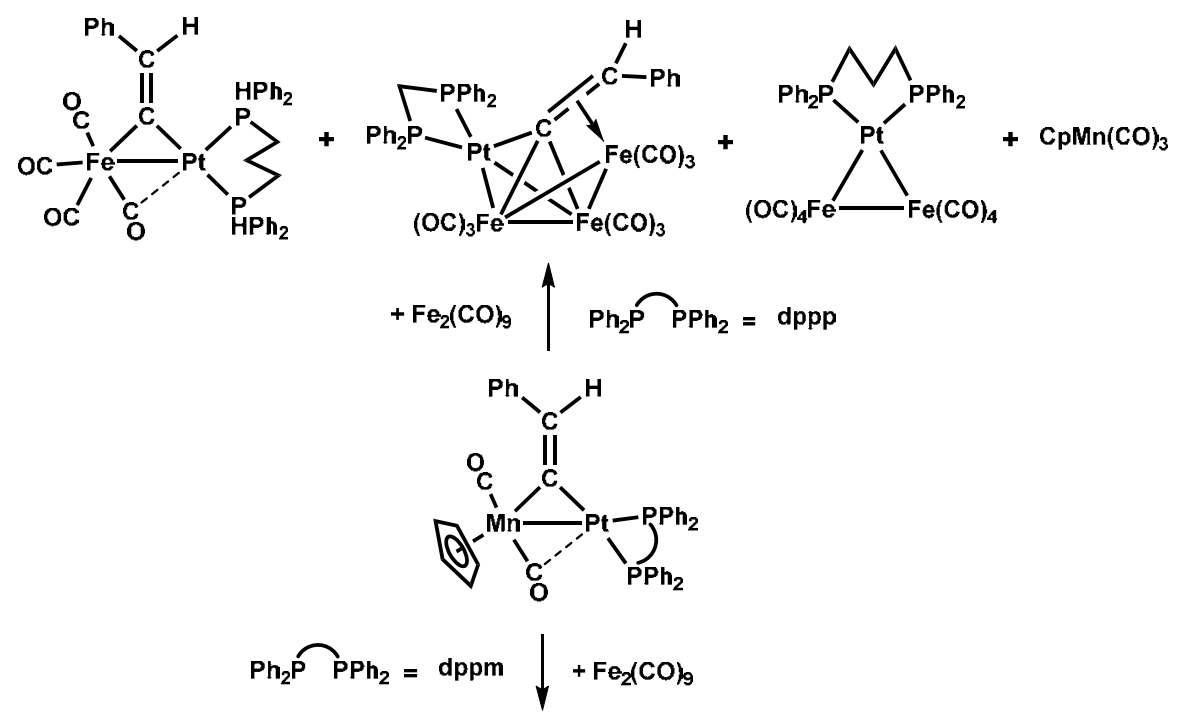

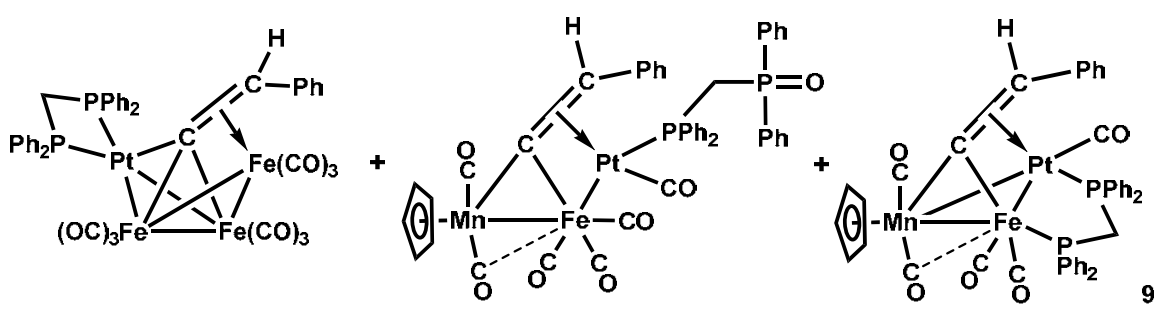

Рис. 1 

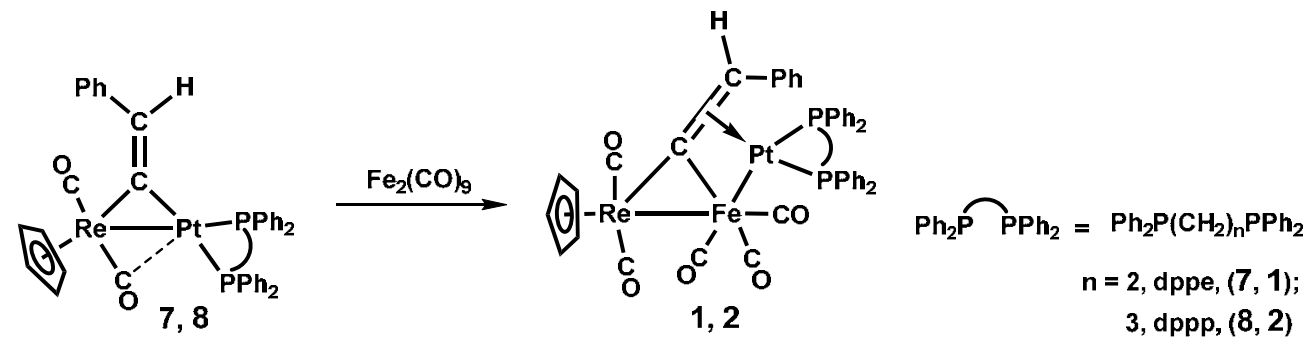

Рис. 2

лиганды путем присоединения третьего металлосодержащего фрагмента к биядерным $\mu$-винилиденовым комплексам, нами были изучены реакции биядерных RePt комплексов $\mathrm{Cp}(\mathrm{CO})_{2} \operatorname{Re}(\mu-\mathrm{C}=\mathrm{CHPh}) \mathrm{Pt}(\mathrm{dppe})(7)$ и $\mathrm{Cp}(\mathrm{CO})_{2} \operatorname{Re}(\mu-\mathrm{C}=\mathrm{CHPh}) \mathrm{Pt}(\mathrm{dppp})(\mathbf{8})$ с нонакарбонилом дижелеза. Показано, что при взаимодействии комплексов 7 и 8 с Fe $2(\mathrm{CO})_{9}$ в бензоле при комнатной температуре получаются новые триметаллические кластеры $\mathrm{CpReFePt}\left(\mu_{3}-\mathrm{C}=\mathrm{CHPh}\right)(\mathrm{CO})_{5}(\mathrm{P}-\mathrm{P})$ (P-P = dppe (1); dppp (2)) с выходами 53 и 41 \% (рис. 2).

Полученные соединения представляют собой темно-красные мелкокристаллические вещества, устойчивые в инертной атмосфере, растворимые в бензоле и хлористом метилене.

Реакция комплексов 7 и 8 с Fe $2(\mathrm{CO})_{9}$ напоминает аналогичную реакцию $\mathrm{RePd}$ комплексов $\mathrm{Cp}(\mathrm{CO})_{2} \operatorname{Re}(\mu-\mathrm{C}=\mathrm{CHPh}) \mathrm{Pd}(\mathrm{dppe})$ и $\mathrm{Cp}(\mathrm{CO})_{2} \operatorname{Re}(\mu-\mathrm{C}=\mathrm{CHPh}) \mathrm{Pd}(\mathrm{dppp})$, однако ReFePt кластеры 1 и 2 в отличие от 5 и 6 [21] не реагируют с $\mathrm{Fe}_{2}(\mathrm{CO})_{9}$, и происходит селективное образование только трехъядерных кластеров, побочных продуктов при этом не наблюдается. Кластеры 1 и 2 по реакциям RePt биядерных комплексов с Fе 2 (CO) $)_{9}$ образуются селективно, но их выход не удается увеличить при использовании больших избытков нонакарбонила дижелеза и увеличении времени реакции. Поэтому для увеличения выходов $\operatorname{CpReFePt}\left(\mu_{3}-\right.$ $\mathrm{C}=\mathrm{CHPh})(\mathrm{CO})_{5}(\mathrm{dppe})(\mathbf{1})$ и $\mathrm{CpReFePt}\left(\mu_{3}-\mathrm{C}=\mathrm{CHPh}\right)(\mathrm{CO})_{5}(\mathrm{dppp})(\mathbf{2})$ нами был опробован другой подход.

Ранее для трехъядерного кластера типа $\mathrm{CpMnFePt}\left(\mu_{3}-\mathrm{C}=\mathrm{CHPh}\right)(\mathrm{CO})_{6}\left[\mathrm{P}\left(\mathrm{OPr}^{\mathrm{i}}\right)_{3}\right]$ была показана возможность замещения карбонильного лиганда при атоме платины на триизопропилфосфитный лиганд с образованием пентакарбонильного кластера $\mathrm{CpMnFePt}\left(\mu_{3}-\mathrm{C}=\mathrm{CHPh}\right)$ $(\mathrm{CO})_{5}\left[\mathrm{P}\left(\mathrm{OPr}^{\mathrm{i}}\right)_{3}\right]_{2}[25]$. Однако возможность замещения как фосфинового, так и карбонильного лагандов при платине в $\mathrm{CpMnFePt}\left(\mu_{3}-\mathrm{C}=\mathrm{CHPh}\right)(\mathrm{CO})_{6}\left[\mathrm{P}(\mathrm{OPr})_{3}\right]$ не была исследована. Известно, что замещение терминальных трифенилфосфиновых лигандов на дифосфины в комплексах $\mathrm{Cp}(\mathrm{CO})_{2} \mathrm{M}(\mu-\mathrm{C}=\mathrm{CHPh}) \operatorname{Pt}\left(\mathrm{PPh}_{3}\right)_{2}(\mathrm{M}=\mathrm{Mn}, \mathrm{Re})$ протекает легко и приводит к образованию биядерных $\mu$-винилиденовых комплексов, содержащих хелатные дифосфиновые лиганды при атоме платины, с количественными выходами $[23,29,30]$. Поэтому нами были изучены реакции $\mathrm{CpReFePt}\left(\mu_{3}-\mathrm{C}=\mathrm{CHPh}\right)(\mathrm{CO})_{6}\left(\mathrm{PPh}_{3}\right)(3)$ с дифосфинами (dppe и dppp). Проведенные эксперименты показали, что при взаимодействии кластера 3 с дифосфином происходит замещение как карбонильного, так и трифенилфосфинового лигандов при атоме платины исходного кластера на хелатный дифосфиновый лиганд. При этом выходы соединений 1 и 2 значительно увеличиваются (87 и 83 \% соответственно) по сравнению с выходами кластеров, полученных по реакции биядерных комплексов 7 и 8 с Fе $2(\mathrm{CO})_{9}$, (рис. 3). 


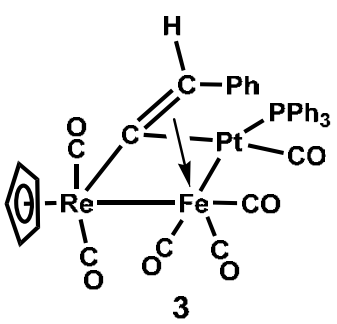

3

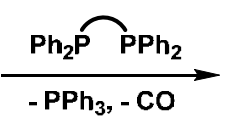

$-\mathrm{PPh}_{3},-\mathrm{CO}$



$\mathrm{Ph}_{2} \mathrm{P} \mathrm{PPh}_{2}=\mathrm{Ph}_{2} \mathrm{P}\left(\mathrm{CH}_{2}\right)_{n} \mathrm{PPh}_{2}$

n = 2, dppe, (1);

3, dppp, (2)

Рис. 3

Таблица. Данные спектров ЯМР ${ }^{1} \mathrm{H},{ }^{31} \mathrm{P}$ и ${ }^{13} \mathrm{C}(\delta, \mathrm{ppm},[\mathrm{J}, \mathrm{Hz}])$ кластеров $\mathrm{CpReFePt}\left(\mu_{3}-\mathrm{C}^{1}=\mathrm{C}^{2} \mathrm{HPh}\right)(\mathrm{CO})_{5}(\mathrm{dppe})$ (1), $\quad \mathrm{CpReFePt}\left(\mu_{3}-\mathrm{C}^{\mathrm{l}}=\mathrm{C}^{2} \mathrm{HPh}\right)(\mathrm{CO})_{5}(\mathrm{dppp}) \quad$ (2), $\mathrm{CpMnFePt}\left(\mu_{3}-\mathrm{C}^{\mathrm{l}}=\mathrm{C}^{2} \mathrm{HPh}\right)(\mathrm{CO})_{5}(\mu-\mathrm{dppm})$ (9) и комплексов $\mathrm{Cp}(\mathrm{CO})_{2} \operatorname{Re}\left(\mu-\mathrm{C}^{\mathrm{l}}=\mathrm{C}^{2} \mathrm{HPh}\right) \mathrm{Pt}(\mathrm{dppe})(7), \mathrm{Cp}(\mathrm{CO})_{2} \operatorname{Re}\left(\mu-\mathrm{C}^{\mathrm{l}}=\mathrm{C}^{2} \mathrm{HPh}\right) \operatorname{Pt}(\mathrm{dppp})(\mathbf{8})$

\begin{tabular}{|c|c|c|c|c|c|c|c|}
\hline \multirow{3}{*}{ Комплекс } & \multicolumn{7}{|c|}{ ЯMP } \\
\hline & \multicolumn{3}{|c|}{${ }^{13} \mathrm{C}$} & \multicolumn{2}{|c|}{${ }^{1} \mathrm{H}$} & \multicolumn{2}{|c|}{${ }^{31} \mathrm{P}$} \\
\hline & $\mathrm{C}^{1}$ & $\mathrm{C}^{2}$ & $\mathrm{C}_{5} \mathrm{H}_{5}$ & $=\mathrm{C}^{2} \underline{\mathrm{H}} \mathrm{Ph}$ & $\mathrm{C}_{5} \mathrm{H}_{5}$ & $\mathrm{P}^{1}$ & $\mathrm{P}^{2}$ \\
\hline 1 & $\begin{array}{l}263,9 \text { д } \\
{ }^{2} J_{\mathrm{CP}}=4 \\
{ }^{*} 255,1 \mathrm{c}\end{array}$ & $\begin{array}{c}96,6 \text { д } \\
{ }^{2} J_{\mathrm{CP}}=29 \\
{ }^{*} 96,3 \text { д } \\
{ }^{2} J_{\mathrm{CP}}=28\end{array}$ & $\begin{array}{l}90,1 \mathrm{c} \\
* 89,4 \mathrm{c}\end{array}$ & $\begin{array}{c}7,01 \text { д } \\
{ }^{3} J_{\mathrm{PtH}}=29 \\
{ }^{4} J_{\mathrm{HP1}}=7,7 \\
{ }^{4} J_{\mathrm{HP} 12}=11,67 \\
{ }^{*} 7,11 \text { д } \\
{ }^{4} J_{\mathrm{HP1}}=7,7\end{array}$ & $\begin{array}{c}5,13 \mathrm{c} \\
* 5,37 \mathrm{c}\end{array}$ & $\begin{array}{c}52,56 \text { д } \\
J_{\mathrm{PtP}}=3300 \\
{ }^{2} J_{\mathrm{PP}}=7,08 \\
{ }^{*} 48,76 \text { д } \\
J_{\mathrm{PtP}}=3300 \\
{ }^{2} J_{\mathrm{PP}}=11,6\end{array}$ & $\begin{array}{c}52,00 \text { д } \\
J_{\mathrm{PtP}}=3600 \\
{ }^{2} J_{\mathrm{PP}}=7,08 \\
{ }^{4} 49,90 \text { д } \\
J_{\mathrm{PtP}}=3500 \\
{ }^{2} J_{\mathrm{PP}}=11,6\end{array}$ \\
\hline 2 & - & - & - & $\begin{array}{c}7,00 \text { дд } \\
{ }^{3} J_{\mathrm{PtH}}=51 \\
{ }^{4} J_{\mathrm{HP} 1}=8,6 \\
{ }^{4} J_{\mathrm{HP} 12}=10,6 \\
{ }^{*} 7,11 \text { д } \\
{ }^{4} J_{\mathrm{HP1}}=4,0\end{array}$ & $\begin{array}{l}5,95 \mathrm{c} \\
{ }^{*} 5,35 \mathrm{c}\end{array}$ & $\begin{array}{c}1,52 \text { д } \\
{ }^{2} J_{\mathrm{PP}}=34,7 \\
J_{\mathrm{PtP}}=3231 \\
{ }^{*}-0,43 \text { д } \\
{ }^{2} J_{\mathrm{PP}}=35,6 \\
J_{\mathrm{PtP}}=3449\end{array}$ & $\begin{array}{c}-0,77 \text { д } \\
{ }^{2} J_{\mathrm{PP}}=34,7 \\
J_{\mathrm{PtP}}=3488 \\
{ }^{*}-1,54 \text { д } \\
{ }^{2} J_{\mathrm{PP}}=35,6 \\
J_{\mathrm{PtP}}=3125\end{array}$ \\
\hline 7 & $\begin{array}{c}234,57 \text { дд } \\
J_{\mathrm{PtCl}}= \\
=764,05 \\
{ }^{2} J_{\mathrm{ClP}}=66,46\end{array}$ & $\begin{array}{c}138,34 \text { дд } \\
{ }^{2} J_{\mathrm{PtC} 2}=101,4 \\
{ }^{3} J_{\mathrm{C} 2 \mathrm{P}}=2,91\end{array}$ & $86,31 \mathrm{c}$ & $\begin{array}{c}8,02 \text { дд } \\
{ }^{3} J_{\mathrm{PtH}}=20,83 \\
{ }^{4} J_{\mathrm{PH}}=13,14, \\
{ }^{4} J_{\mathrm{PH}}=14,29\end{array}$ & $5,09 \mathrm{c}$ & $\begin{array}{c}49,20 \text { д } \\
{ }^{2} J_{\mathrm{PP}}=36,34 \\
J_{\mathrm{PtP}}=4267\end{array}$ & $\begin{array}{c}51,89 \text { д } \\
{ }^{2} J_{\mathrm{PP}}=36,34 \\
J_{\mathrm{PtP}}=2493\end{array}$ \\
\hline 8 & $\begin{array}{c}233,41 \text { дд } \\
J_{\mathrm{PtCl}}=813,79 \\
{ }^{2} J_{\mathrm{ClP}}=65,55 ; \\
2,59\end{array}$ & $\begin{array}{c}137,61 \mathrm{~T} \\
{ }^{2} J_{\mathrm{PtC} 2}= \\
=104,99\end{array}$ & $85,61 \mathrm{c}$ & $\begin{array}{c}7.17 \text { дд } \\
{ }^{3} J_{\mathrm{PtH}}=17,66 \\
{ }^{4} J_{\mathrm{PH}}=13,19 \\
{ }^{4} J_{\mathrm{PH}}=15,42\end{array}$ & $4,82 \mathrm{c}$ & $\begin{array}{c}14,77 \text { д } \\
{ }^{2} J_{\mathrm{PP}}=6,24 \\
J_{\mathrm{PtP}}=4285\end{array}$ & $\begin{array}{c}9,90 \text { д } \\
{ }^{2} J_{\mathrm{PP}}=6,24 \\
J_{\mathrm{PtP}}=2312\end{array}$ \\
\hline 9 & $\begin{array}{c}311,70 \mathrm{c} \\
J_{\mathrm{PtCl} 1}=68,5\end{array}$ & $\begin{array}{c}94,17 \text { д } \\
{ }^{2} J_{\mathrm{PtC} 2}=169,6 \\
{ }^{3} J_{\mathrm{PC} 2}=3,4\end{array}$ & $\begin{array}{l}88,25 \mathrm{c} \\
{ }^{*} 88,46 \mathrm{c}\end{array}$ & $\begin{array}{c}7,01 \text { д } \\
{ }^{3} J_{\mathrm{PtH}}=55,1 \\
{ }^{4} J_{\mathrm{PH}}=3,0 \\
{ }^{*} 6,23 \mathrm{c} \\
{ }^{3} J_{\mathrm{PtH}}=54,3 \\
{ }^{4} J_{\mathrm{PH}}=3,0\end{array}$ & $\begin{array}{l}4,49 \mathrm{c} \\
* 4,80 \mathrm{c}\end{array}$ & $\begin{array}{c}17,84 \text { д } \\
J_{\mathrm{PtP}}=3439 \\
{ }^{2} J_{\mathrm{PP}}=31 \\
{ }^{*} 12,89 \text { д } \\
J_{\mathrm{PtP}}=3346 \\
{ }^{2} J_{\mathrm{PP}}=29\end{array}$ & $\begin{array}{c}65,96 \text { д } \\
J_{\mathrm{PtP}}=96 \\
{ }^{2} J_{\mathrm{PP}}=31 \\
{ }^{6} 68,74 \text { д } \\
J_{\mathrm{PtP}}=58 \\
{ }^{2} J_{\mathrm{PP}}=29\end{array}$ \\
\hline
\end{tabular}

Примечание * - химические сдвиги изомеров кластеров.

Полученные соединения изучены методами ИК-, ЯМР-спектроскопии. В таблице приведены основные данные спектров ЯМР ${ }^{1} \mathrm{H},{ }^{31} \mathrm{P}$ и ${ }^{13} \mathrm{C}$ кластеров 1 и 2.

Из данных ЯМР-спектров полученных соединений (таблица) видно, что кластеры 1 и 2 в растворах находятся в виде смеси двух изомеров. На основе измерения соотношений инте- 
гральных интенсивностей сигналов атомов фосфора в спектрах ЯМР ${ }^{31} \mathrm{P}$ и сигналов циклопентадиенильных лигандов в спектрах ЯМР ${ }^{1} \mathrm{H}$ кластеров установлено, что соотношение изомеров составляет 3:1 и 1:1 для кластеров 1 и 2 соответственно.

O наличии в полученных кластерах мостикового $\mu_{3}-\eta^{2}: \eta^{1}: \eta^{1}$-координированного винилиденового лиганда однозначно свидетельствуют данные спектров ЯМР ${ }^{1} \mathrm{H}$ и ${ }^{13} \mathrm{C}$, в которых сигналы винилиденового и циклопентадиенильного лигандов находятся в областях $\delta$ типичных для $\mu_{3}$-винилиденовых трехъядерных кластеров (табл. 1) $[11,26]$. Однако в ЯМР-спектрах синтезированных кластеров 1 и 2 обнаружены некоторые особенности, которые дают возможность лучше понять их строение.

Так, например, при переходе от исходного биядерного комплекса $\mathrm{Cp}(\mathrm{CO})_{2} \operatorname{Re}(\mu-\mathrm{C}=\mathrm{CHPh})$ $\mathrm{Pt}(\mathrm{dppe})(7)$ к соответствующему кластеру $\mathrm{CpReFePt}\left(\mu_{3}-\mathrm{C}=\mathrm{CHPh}\right)(\mathrm{CO})_{5}(\mathrm{dppe})(\mathbf{1})$ в спектре ЯМР ${ }^{13} \mathrm{C}$ наблюдается не только смещение химических сдвигов атомов $\mathrm{C}^{1}$ и $\mathrm{C}^{2}$ винилиденового лиганда, но также изменение величины их констант $J_{\text {СР }}$ (таблица). В спектре ЯМР ${ }^{13} \mathrm{C}$ кластера 1 константа спин-спинового взаимодействия у атома $\mathrm{C}^{2}$ в несколько раз больше, чем у атома $\mathrm{C}^{1}$, в исходном комплексе 7 с мостиковым $\mu_{2}$-винилиденовым лигандом, и наоборот, константа ${ }^{2} J_{\mathrm{CP}}$ атома $\mathrm{C}^{1}$ в несколько раз больше константы ${ }^{3} J_{\mathrm{CP}}$ aтома $\mathrm{C}^{2}$ (таблица). Такое изменение значений констант $J_{\mathrm{CP}}$ атомов $\mathrm{C}^{1}$ и $\mathrm{C}^{2}$ свидетельствует о $\pi$-координации винилиденового лиганда с атомом платины в 1. Ранее подобное изменение констант $J_{\mathrm{CP}}$ наблюдалось при переходе от биядерного $\operatorname{RePd}$ комплекса к ReFePd трехъядерному кластеру $\mathbf{5}$, в котором согласно рентгеноструктурным данным винилиденовый лиганд образует $\sigma$-связи с атомами рения и железа и $\pi$-связь с атомом палладия [21].

В исходных биядерных комплексах 7 и 8 атомы фосфора дифосфиновых лигандов неэквивалентны и находятся в разных положениях относительно $\mu$-винилиденового лиганда $[23,30]$. Эта неэквивалентность отражается в их спектрах ЯМР ${ }^{31}$ Р. Так, сигнал в слабом поле, имеющий большую величину константы $J_{\mathrm{PtP}}$, был отнесен к атому $\mathrm{P}^{1}$, находящемуся в цис-положении к винилиденовому лиганду, а сигнал в сильном поле со значительно меньшей константой $J_{\mathrm{PtP}}$ к атому $\mathrm{P}^{2}$ в транс-положении к винилидену. В спектрах ЯМР ${ }^{31} \mathrm{P}$ трехъядерного кластера $\mathrm{CpMnFePt}\left(\mu_{3}-\mathrm{C}=\mathrm{CHPh}\right)(\mathrm{CO})_{5}(\mu$-dppm) (9) найдено, что разница между химическими сдвигами ядер $\mathrm{P}^{1}$ и $\mathrm{P}^{2}$ лиганда dppm составляла 50 м.д., а константа $J_{\mathrm{PtP}}$ атома фосфора, координированного с железным фрагментом, была в 60 раз меньше константы $J_{\mathrm{PtP}}$ атома фосфора, координированого с платиной (таблица) [28]. Эти данные свидетельствуют о том, что в 9 один фосфор лиганда dppm координирован с атомам платины, а другой - с атомом железа. Подобные сдвиги атомов фосфора и константы $J_{\mathrm{PtP}}$ обнаружены и для других соединений, в которых хелатные дифосфины связывают атомы железа и платины [31, 32]. В спектрах ЯМР ${ }^{31}$ Р кластеров 1 и 2 также наблюдаются неэквивалентные сигналы ядер фосфора (таблица), что свидетельствует о том, что оба атома фосфора дифосфинового лиганда связаны с платиновым фрагментом.

Таким образом, показано, что кластеры $\mathrm{CpReFePt}\left(\mu_{3}-\mathrm{C}=\mathrm{CHPh}\right)(\mathrm{CO})_{5}(\mathrm{dppe})$ (1) и $\mathrm{CpReFePt}\left(\mu_{3}-\mathrm{C}=\mathrm{CHPh}\right)(\mathrm{CO})_{5}(\mathrm{dppp})(2)$ могут быть синтезированы с помощью двух разных подходов. Первый метод основан на металлировании биядерных винилиденовых комплексов 7 и $\mathbf{8}$ в ходе которого происходит координация фрагмента $\left[\mathrm{Fe}(\mathrm{CO})_{3}\right]$ по связи $\mathrm{C}=\mathrm{C}$ винилиденового лиганда исходного комплекса, при этом образуется триметаллический остов ReFePt, a винилиденовый лиганд приобретает мостиковую $\mu_{3}-\eta^{2}: \eta^{1}: \eta^{1}$-конфигурацию. Второй метод состоит в 
модификации лигандного окружения известного ReFePt кластера 3, при этом идет замещение карбонильного и трифенилфосфинового лигандов в платинасодержащем фрагменте исходного кластера на хелатный дифосфиновый лиганд, конфигурация металлоостова не изменяется. На основании анализа данных спектров ИК и ЯМР ${ }^{1} \mathrm{H},{ }^{31} \mathrm{P}$ и ${ }^{13} \mathrm{C}$ и сравнения их с данными ранее синтезированных би- и трехъядерных соединений предложено строение трехъядерных кластеров 1 и 2. Установлено, что винилиденовый лиганд в этих соединениях координирован с тремя атомами металлов, образуя $\sigma$-связи с атомами рения и железа и $\pi$-связь с атомом платины; оба атома фосфора дифосфиновых лигандов связаны с платиновым фрагментом.

Работа выполнена в рамках комплексной программы фундаментальных исследований Сибирского отделения РАН № II.2 «Интеграция и развитие», проект № II.2П.V44-13 "Синтез и исследование свойств гетерометаллических трехъядерных винилиденовых кластеров - перспективных предщественников гетерометаллических материалов и новых химических веществ».

\section{Список литературы}

1. Sculfort S., Braunstein P. Intramolecular d10-d10 interactions in heterometallic clusters of the transition metals. Chem. Soc. Rev. 2011. Vol. 40(5). P. 2741-2760.

2. Adams R.D. Metal segregation in bimetallic clusters and its possible role in synergism and bifunctional catalysis. J. Organomet Chem. 2000. Vol. 600 (1-2). P. 1-6.

3. Xiao J., Puddephatt R.J. Pt-Re clusters and bimetallic catalysts. Coord. Chem. Rev. 1995. Vol. 143. P. 457-500.

4. Ungva F. Application of transition metals in hydroformylation. Annual survey covering the year 2000. Coord. Chem. Rev. 2001. Vol. 218. P. 1-41.

5. Thomas J.M. et al. High-performance nanocatalysts for single-step hydrogenations. Acc. Chem. Res. 2003. Vol. 36, № 1. P. 20-30.

6. Werner H. Vinylidenerhodium complexes as promising tools for $\mathrm{C}-\mathrm{C}$ coupling reactions Coord. Chem. Rev. 2004. Vol. 248, № 15-16. P. 1693-1702.

7. Ritleng V., Chetcuti M.J. Hydrocarbyl ligand transformations on heterobimetallic complexes. Chem. Rev. 2007. Vol. 107(3). P. 797-858.

8. Wheatley N., Kalck P. Structure and reactivity of early-late heterobimetallic complexes. Chem. Rev. 1999. Vol. 99(1). P. 3379-3419.

9. Adams R.D., Captain B. Hydrogen activation by unsaturated mixed-metal cluster complexes: new directions. Angew. Chem. Int. Ed. Engl. 2008. Vol. 47, № 2. P. 252-257.

10. Able E.W., Stone F.G.A., Wilkinson G. Comprehensive Organometallic Chemistry: Heteronuclear metal-metal bonds. Vol. 10. Under ed. Adams R.D. Pergamon, 1995. 423 p.

11. Bruce M.I. Organometallic chemistry of vinylidene and related unsaturated carbenes. Chem. Rev. 1991. Vol. 91(2). P. 197-257.

12. Zhang L. et al. Remarkable Reactions of Cationic Carbyne Complexes of Manganese and Rhenium with the Carbonylrhodium Anion $\left[\mathrm{Rh}(\mathrm{CO})_{4}\right]^{-}$. A Novel Route to Heteropolymetallic Bridging Carbyne Complexes with $\mu$ - and $\mu_{3}$-Carbyne Ligands. Organometallics. 2003. Vol. 22(5). P. 4369-4371. 
13. Werner H. et al. The Rhodium Compounds $\mathrm{C}_{5} \mathrm{H}_{5} \mathrm{Rh}(=\mathrm{C}=\mathrm{CHR}) \mathrm{P}^{\mathrm{P}} \mathrm{Pr}_{3}$ as Building Blocks for the Synthesis of Heterometallic Di- and Trinuclear Vinylidene-Bridged Complexes. Chem. Ber. 1988. Vol. 121. P. 1565-1573.

14. Berenguer J.R., Lalinde E., Teresa Moreno M. An overview of the chemistry of homo and heteropolynuclear platinum complexes containing bridging acetylide $(\mu-\mathrm{C} \equiv \mathrm{CR})$ ligands. Coord. Chem. Rev. 2010. Vol. 254(7-8). P. 832-875.

15. Stone F.G.A. Zero-valent platinum complexes and their role in the synthesis of di-, tri-, and tetra-nuclear metal compounds. Inorg. Chim. Acta. 1981. Vol. 50. P. 33-42.

16. Bruce M.I. Transition Metal Complexes Containing Allenylidene, Cumulenylidene, and Related Ligands. Chem. Rev. 1998. Vol. 98(8). P. 2797-2858.

17. Werner H. et al. Ambidentate Behavior of Mononuclear Vinylidenerhodium Complexes - Novel C-C Coupling of a Methyl to a Vinylidene Group. Angew. Chem. Int. Ed. Engl. 1984. Vol. 23. P. 431-432.

18. Kolobova N.E. et al. Reactions of manganese $\pi$-acetylenic, $\eta$-vinylidenic, and $\eta$-allenylidenic complexes with $\mathrm{Fe}_{2}(\mathrm{CO})_{9}$ crystal and molecular structure of $\mathrm{Cp}(\mathrm{CO})_{2} \mathrm{Mn}\left(\mu_{2}-\mathrm{C}=\mathrm{CHCOOCH}_{3}\right) \mathrm{Fe}(\mathrm{CO})_{4}$. J. Organomet. Chem. 1982. Vol. 228. P. 265-272.

19. Werner H. et al. Synthese und Kristallstruktur von Heterometall-zweikernkomplexen mit Vinyliden-brückenliganden. J. Organomet. Chem. 1985. Vol. 289. P. C5-C12.

20. Antonova A.B. et al. Chemistry of vinylidene complexes. XVIII. Synthesis and molecular structure of the novel trinuclear $\mu 3$-vinylidene complex $\mathrm{CpReFePt}\left(\mu_{3}-\mathrm{C}=\mathrm{CHPh}\right)(\mathrm{CO})_{6}\left(\mathrm{PPh}_{3}\right) . J$. Organomet. Chem. 2009. Vol. 694(1). P. 127-130.

21. Verpekin V.V. et al. Chemistry of vinylidene complexes. XXIII. Binuclear rhenium-palladium vinylidene bridged complexes, their reactions with diiron nonacarbonyl. J. Organomet. Chem. 2014. Vol. 770. P. 42-50.

22. Верпекин В.В., Чудин О.С., Пирязев Д.А., Рубайло А.И., Громилов С.А., Семейкин О.В. Новый трехъядерный $\mu_{3}-$ винилиденовый кластер $\mathrm{CpReFePt}\left(\mu_{3}-\mathrm{C}=\mathrm{CHPh}\right)(\mathrm{CO})_{5}\left[\mathrm{P}\left(\mathrm{OPr}^{\prime}\right)_{3}\right]_{2}$. Жур нал структурной химии. 2015. Vol. 44. P. 823-825.

23. Antonova A.B. et al. Chemistry of vinylidene complexes. XXI. Synthesis, spectroscopic and structural study of the RePt and MnPt $\mu$-vinylidene complexes. Inorg. Chim. Acta. 2013. Vol. 394. P. 328-336.

24. Braye E.H. et al. Diiron Enneacarbonyl. John Wiley \& Sons, Inc. P. 178-181.

25. Antonova A.B. et al. Chemistry of vinylidene complexes XI. Synthesis of trinuclear MnFePt complexes by means of consecutive assemblig out of mono- and dimetal vinylidene precursors. Inorganica Chim. Acta. 1995. Vol. 230. P. 97-104.

26. Antonova A.B. Use of the $\mathrm{Mn}=\mathrm{C}=\mathrm{C}$ system in organometallic and organic synthesis Coord. Chem. Rev. 2007. Vol. 251(11-12). P. 1521-1560.

27. Antonova A.B. et al. Chemistry of vinylidene complexes XII. Transmetalation of the ixvinylidene ligand in the reaction of $\mathrm{Cp}(\mathrm{CO})_{2} \mathrm{MnPt}(\mu-\mathrm{C}=\mathrm{CHPh})(\mathrm{dppp})$ with $\mathrm{Fe}_{2}(\mathrm{CO})_{9}$. Formation of new Pt, $\mathrm{PtFe}_{2}$ and $\mathrm{PtFe}_{3}$ complexes. J. Organomet. Chem. 1996. Vol. 524. P. 81-85.

28. Antonova A.B. et al. Chemistry of vinylidene complexes. XIII. The reaction between $\mathrm{Cp}(\mathrm{CO})_{2} \mathrm{MnPt}(\mu-\mathrm{C}=\mathrm{CHPh})\left(\eta^{2}-\mathrm{dppm}\right)$ and $\mathrm{Fe}_{2}(\mathrm{CO})_{9}$ : simultaneous formation of the $\mu_{3}$-vinylidene $\mathrm{MnFePt}$ and $\mu_{4}$-vinylidene $\mathrm{PtFe}_{3}$ clusters . Crystal structure of $\left(\eta^{5}-\mathrm{C}_{5} \mathrm{H}_{5}\right) \mathrm{MnFePt}\left(\mu_{3}-\mathrm{C}=\mathrm{CHPh}\right)$ (CO) ${ }_{6}\left[\eta^{1}-\mathrm{PPh}_{2} \mathrm{CH}_{2} \mathrm{P}(=\mathrm{O}) \mathrm{Ph}_{2}\right.$. J. Organomet. Chem. 1999. Vol. 577. P. 238-242. 
29. Antonova A.B. et al. Chemistry of vinylidene complexes X. Synthesis and characterization of the vinylidene bridged complexes $\mathrm{Cp}(\mathrm{CO}){ }_{2} \mathrm{MnPt}(\mu-\mathrm{C}=\mathrm{CHPh})(\mathrm{P}-\mathrm{P})$ with chelating diphosphine ligands $\mathrm{P}-\mathrm{P}=\mathrm{dppm}$, dppe or dppp at the platinum atom. Inorganica Chim. Acta. 1991. Vol. 182. P. 49-54.

30. Антонова А.Б. et al. Химия винилиденовых комплексов. Сообщение 19 . Новые гетерометаллические $\mu$-винилиденовые комплексы со связями $\mathrm{Re}-\mathrm{M}(\mathrm{M}=\mathrm{Pd}, \mathrm{Pt})$. Молекулярная структура $\left(\eta^{5}-\mathrm{C}_{5} \mathrm{H}_{5}\right)(\mathrm{CO}) \operatorname{RePt}(\mu-\mathrm{C}=\mathrm{CHPh})(\mu-\mathrm{CO})\left(\mathrm{Ph}_{2} \mathrm{PCH}_{2} \mathrm{PPh}_{2}\right)$. Известия Академии наук. Серия химическая. 2009. Vol. 5. P. 933-940.

31. Knorr M., Strohmann C., Braunstein P. Reactivity of Silylated Dinuclear Iron-Platinum Acyl Complexes: Formation of $\mu$-Vinylidene Complexes and Crystal Structures of the Acyl Complex $\left[(\mathrm{OC})_{3}\left\{(\mathrm{MeO})_{3} \mathrm{Si}\right\} \mathrm{Fe}(\mu-\mathrm{dppm}) \mathrm{Pt}\{\mathrm{C}(\mathrm{O}) \mathrm{Me}\}(\mathrm{t}-\mathrm{BuNC})\right]$ and the $\mu$-Vinylidene Complex $\left[(\mathrm{OC})_{3} \mathrm{Fe}\{\mu-\mathrm{C}=\mathrm{C}(\mathrm{H}) \mathrm{Ph}\}(\mu-\mathrm{dppm}) \mathrm{Pt}\left(\mathrm{PPh}_{3}\right)\right]$. Organometallics. 1996. Vol. 15, № 6. P. 5653-5663.

32. Braunstein B.P. et al. Mixed-Metal Cluster Formatiom By Carbonylmetalate-Induced Pt-P Bond Cleavage in $\left[\mathrm{PtCl}_{2}\left(\mathrm{Ph}_{2} \mathrm{PCH}_{2} \mathrm{PPh}_{2}\right)\right]$. Angew. Chem. Int. Ed. Engl. 1987. Vol. 26. P. 88-89. 